\title{
Histopathological findings in cochlear implants in cats
}

\author{
By G. M. Clark, H. G. Kranz, H. Minas and J. M. Nathar \\ (Melbourne, Australia)
}

\section{Introduction}

IF cochlear implants are to be used on patients, it is important that experimental studies should be carried out on animals so that tissue tolerance and other long term effects of electrode implantations can be assessed. Consequently, an experimental study by Simmons ( 1967 ) is of interest, as it has shown that a stainless steel electrode inserted into the basal turn of the cochlea through the round window can be tolerated, and not lead to permanent damage unless infection supervenes. Furthermore, in a study by Axelsson and Hallén (1973) it has been demonstrated that drilling an opening in the cochlea will usually only lead to localized damage of the cochlear structures, and that the opening in the bone normally heals.

In the present study, the tissue damage following the implantation of electrodes into the cochlea through the round window or an opening drilled over the apical and middle turns has been examined and compared.

This comparison is important, as it can be difficult to introduce an electrode array into the middle and apical turns through the round window, and as it may be desirable to implant electrodes in these turns in certain types of deafness, this will have to be done by drilling an opening in the overlying bone (Clark, I975).

\section{Methods}

Electrodes were implanted in nine adult cats. The operations were carried out in surgically clean conditions, and the anaesthetic was induced with pentobarbitone sodium ( $40 \mathrm{mg} / \mathrm{kg}$, ip) and followed by methoxyfluorane and halothane administered by a closed circuit technique. The cats were given procaine penicillin $(500,000$ units, imi) or chloromycetin succinate (I $25 \mathrm{mg}$, imi) during the operation, and for 4 to 8 days postoperatively. The electrodes used were enamelled stainless steel (outside diameter, $0.125 \mathrm{~mm}$ ), trimel-coated silver (outside diameter, $0.15 \mathrm{~mm}$ ), and trimel-coated platinum (outside diameter, $0 \cdot 157 \mathrm{~mm}$ ). Electrical stimulation was carried out at currents which varied from 50-500 $\mu$ amp. 


\section{G. M. Clark et al.}

After time intervals which varied from two to six months, the animals were sacrificed, and perfused intra-arterially with normal saline followed by ro per cent formalin. The temporal bones were then removed, decalcified, embedded in celloidin, sectioned and stained with haematoxylin and eosin.

\section{Cat 39-72.}

\section{Results}

A bipolar stainless steel electrode was inserted through the round window, penicillin was administered post-operatively, the temporal bone was irradiated with $\mathrm{r}, 300$ rads to destroy the hair cells, and the animal perfused intra-arterially five months following the implantations.

The histopathology (Fig. I) showed complete loss of hair cells and

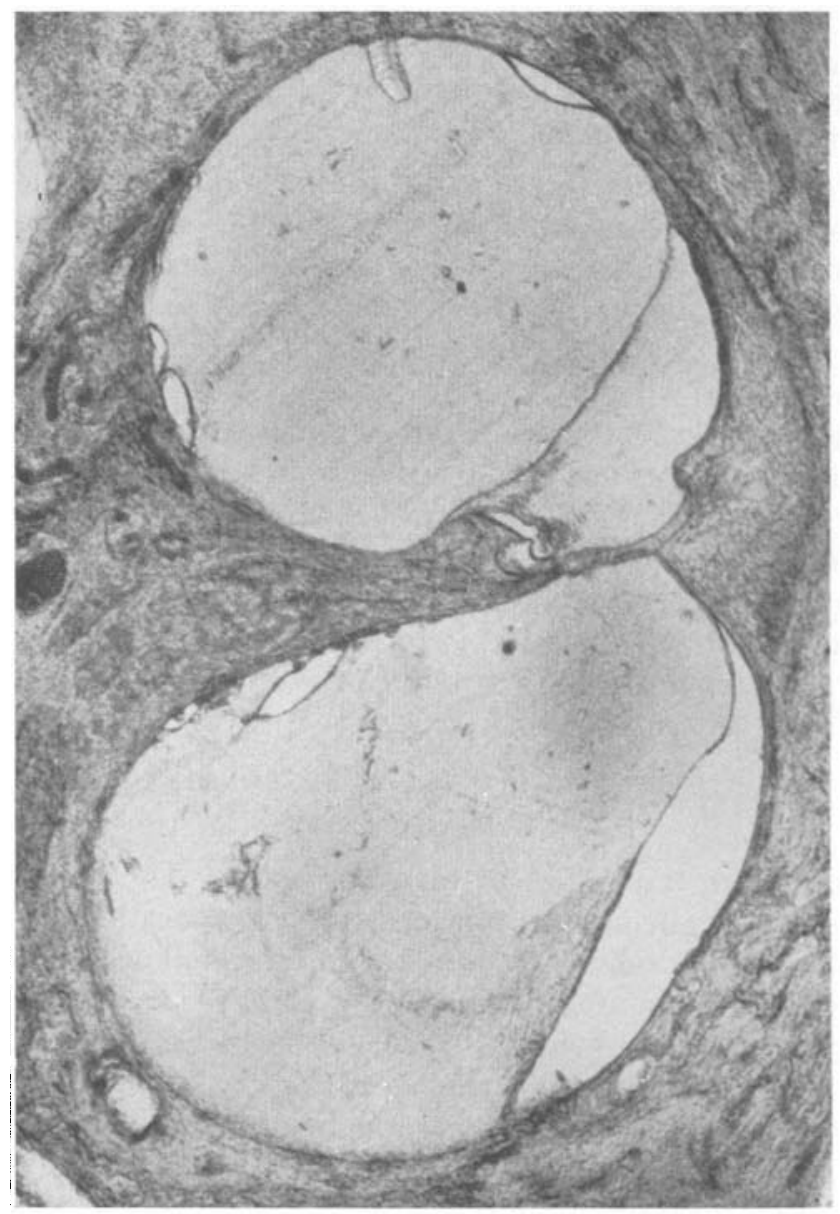

FIG. I.

Photomicrograph of the basal turn in Cat $39-72$.

496 


\section{Histopathological findings in cochlear implants in cats}

atrophy of the tectorial membrane and organ of Corti in all turns. The spiral ganglion cells, however, were not reduced in numbers, and the auditory nerve fibres were intact.

Cat $\mathrm{I}-73$.

A bipolar platinum electrode was inserted through the round window, kanamycin solution was instilled to destroy the hair cells, penicillin was administered post-operatively, and the animal was perfused intraarterially three months following the implantation.

The histopathology (Figs. $2 a$ and $2 b$ ) showed a loss of the organ of Corti in all turns, and there was atrophy of most of the ganglion cells and degenerative changes in the auditory nerve. It is also interesting to note the marked thickening of Reissner's membrane in the middle and apical turns, and the fibrous tissue and bone formation seen in the scala tympani of the basal turn (Fig. $2 b$ ).

Cat $40-72$.

A bipolar silver electrode was inserted through the round window, penicillin was administered post-operatively, the temporal bone was irradiated with I,300 rads to destroy the hair cells, and the animal was perfused intra-arterially four months after the implantation.

The histopathology showed loss of the organ of Corti and Reissner's membrane in all cochlear turns, and there was atrophy of about one-third of the spiral ganglion cells. The scala tympani in the basal and middle turns were filled with loose fibrous tissue and deposits of silver.

Cat $9-72$.

Bipolar stainless steel electrodes were inserted directly into the apical and basal turns, penicillin was administered post-operatively, and the animal sacrificed two months after the implantation.

Histopathology (Fig. 3) showed complete loss of hair cells in the apical and middle turns, and there was destruction of the organ of Corti in the basal turn where the scala tympani and scala media were replaced with fibrous tissue. It was interesting to note that there was atrophy of only a small number of spiral ganglion cells, and the auditory nerve fibres appeared intact.

Cat $5-72$.

Bipolar stainless steel electrodes were inserted directly into the apical and basal turns, penicillin was administered post-operatively, and the animal was perfused intra-arterially five months after the implantation.

Histopathology (Figs. $4 a$ and $4 b$ ) showed there was marked atrophy of the organ of Corti in the middle and apical turns. In the basal turn (Fig. $4 b$ ) there was destruction of the organ of Corti, and there were pus cells under the endothelial lining of the scala vestibuli. There was loss of a third of the ganglion cells, but the auditory nerve fibres appeared intact. 


\section{G. M. Clark et al.}

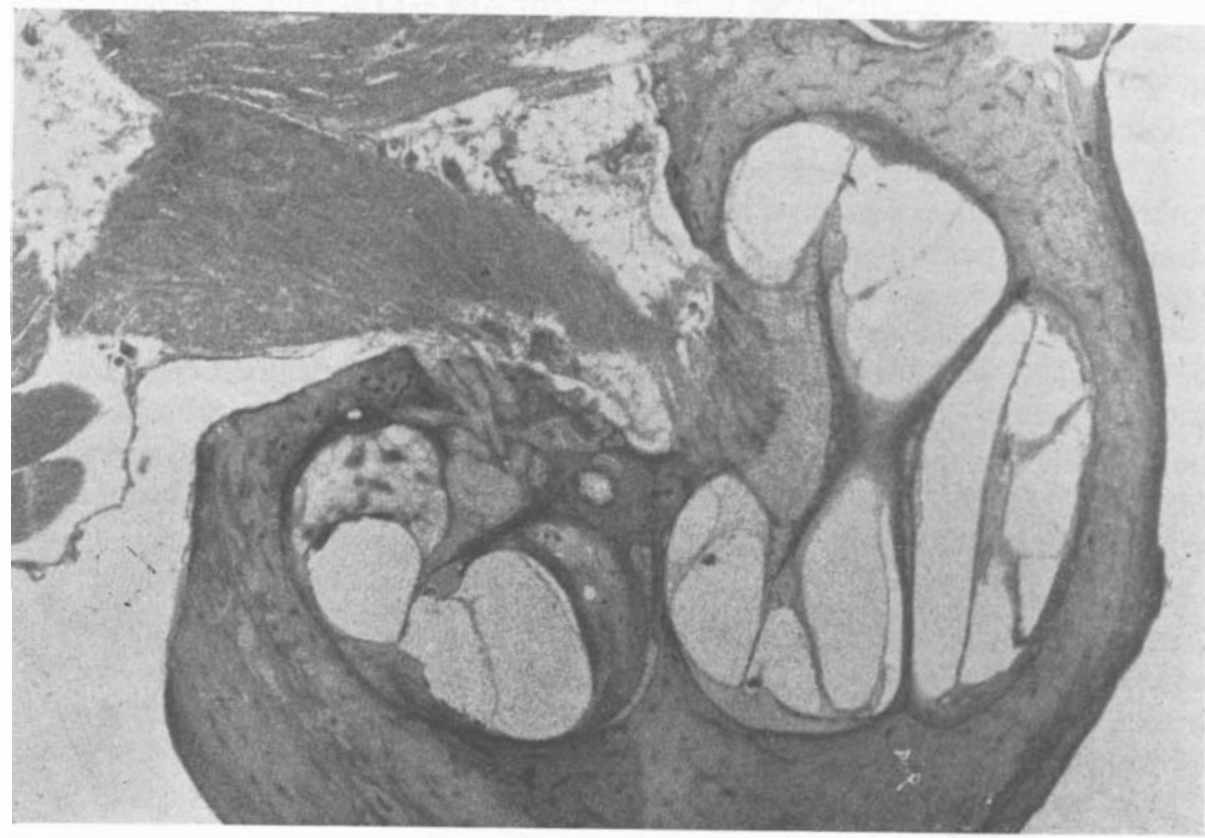

î.

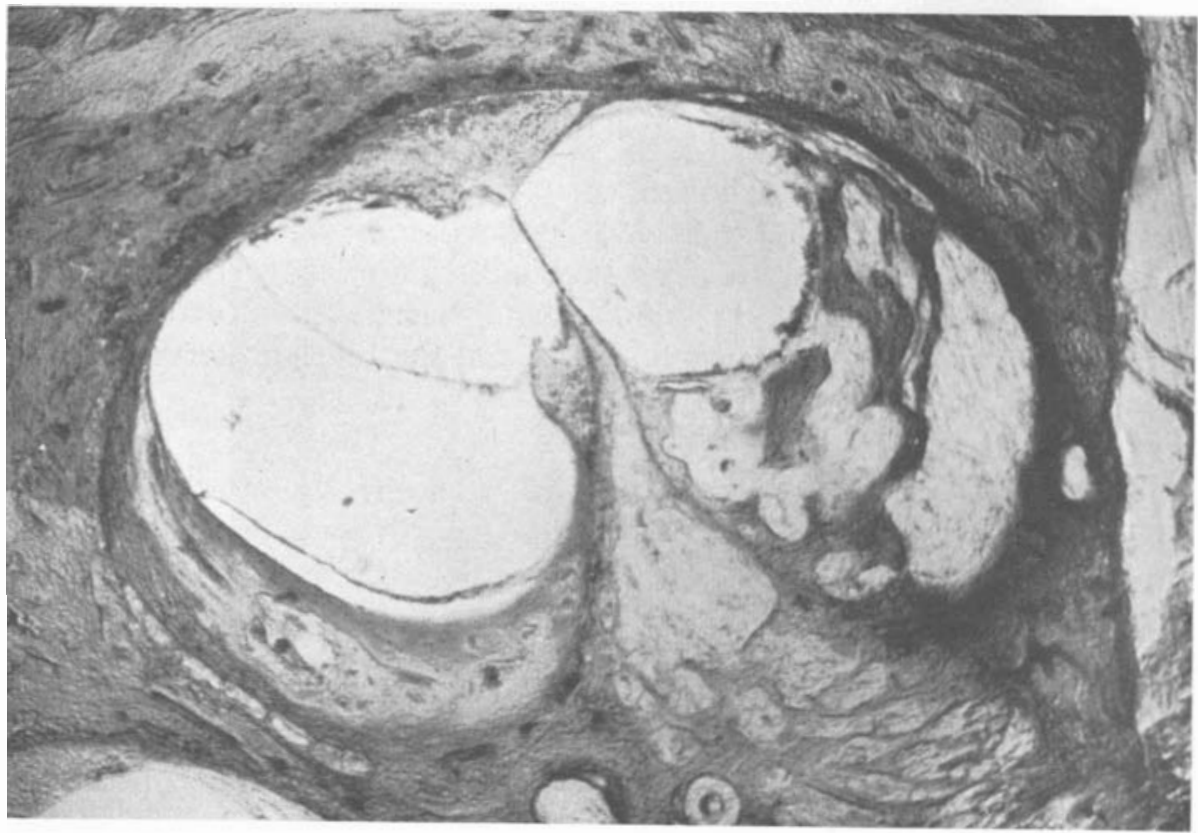

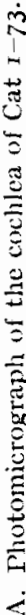




\section{Histopathological findings in cochlear implants in cats}

\section{Cat 4-72.}

Bipolar stainless steel electrodes were inserted directly into the apical and basal turns. Penicillin was administered post-operatively, and the animal was perfused intra-arterially six months after the implantation.

Histopathology showed atrophy of the organ of Corti in all the turns, and there was exudate in the scalae with the formation of loose areolar tissue in the scala tympani of the basal turn (Fig. 5). There was no loss in the number of ganglion cells, and the auditory nerve fibres were intact.

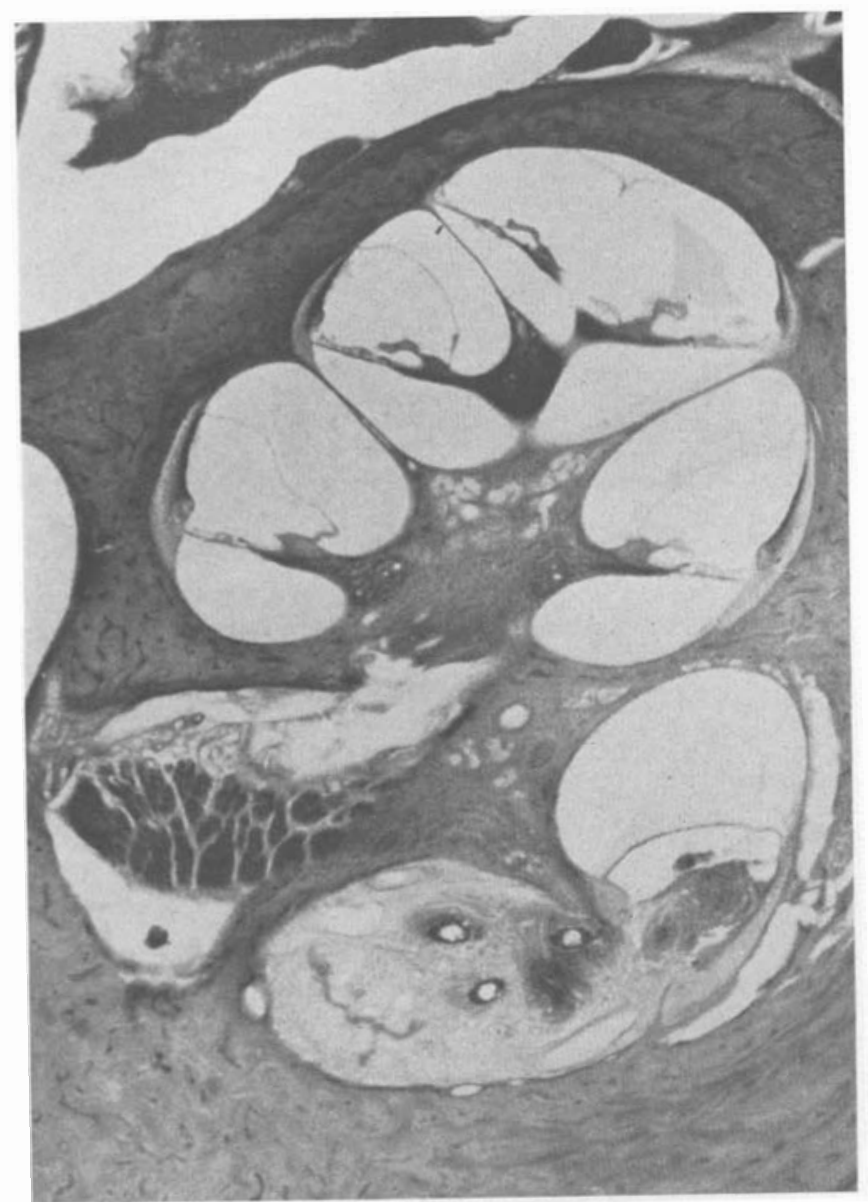

FIG. 3 .

Photomicrograph of the cochlea of Cat 9-72.

Cat $4 \mathrm{I}-72$.

Bipolar stainless steel electrodes were inserted directly into the apical and basal turns of the cochlea. Penicillin was administered post- 
G. M. Clark et al.
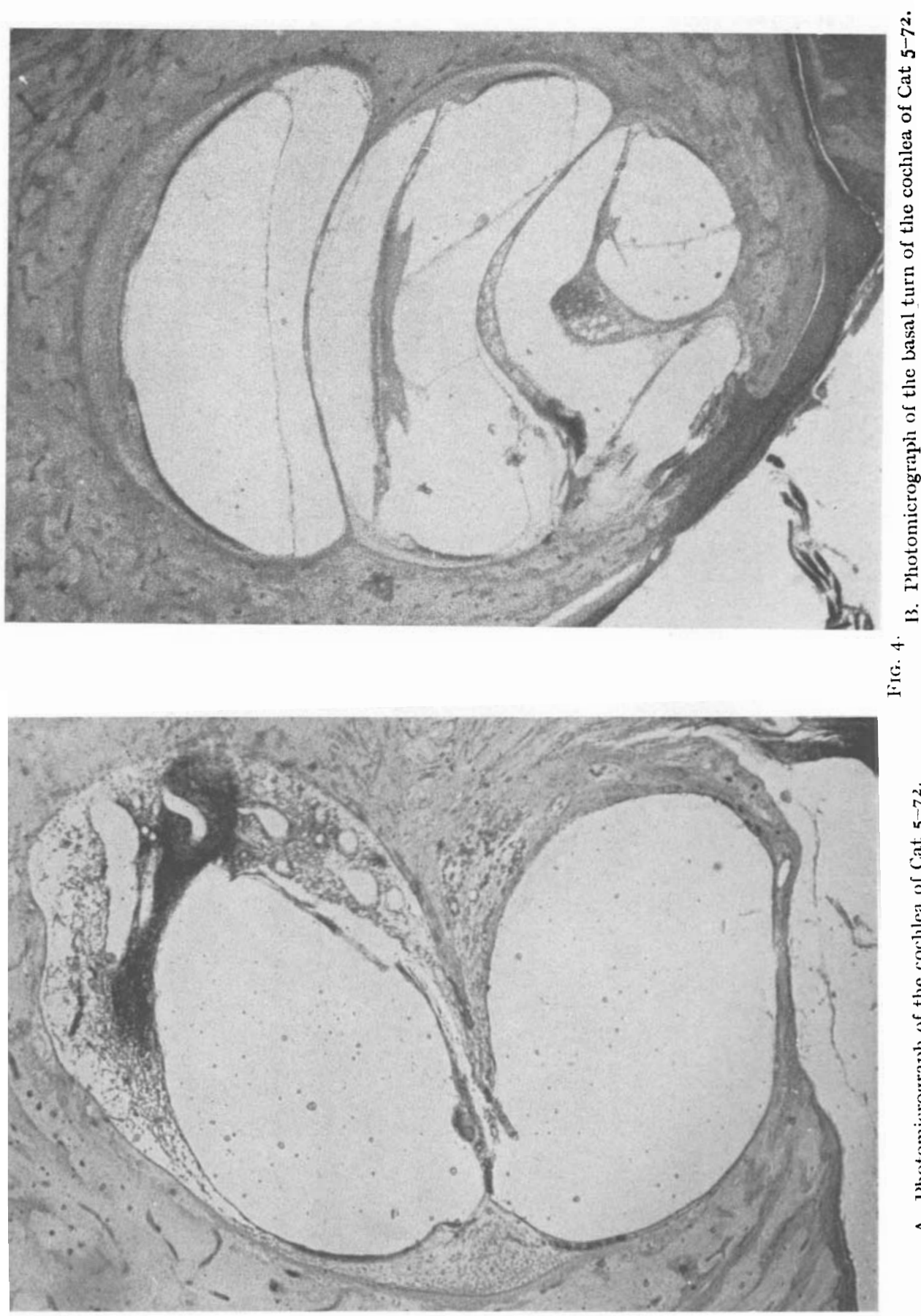

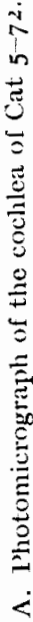




\section{Histopathological findings in cochlear implants in cats}

operatively, and the animal was perfused intra-arterially two months after implantation.

Histopathology showed the loss of the organ of Corti and Reissner's membrane in all the turns, and the scala tympani was filled with fibrous tissue. There was loss of the spiral ganglion cells, and atrophy of the auditory nerve.

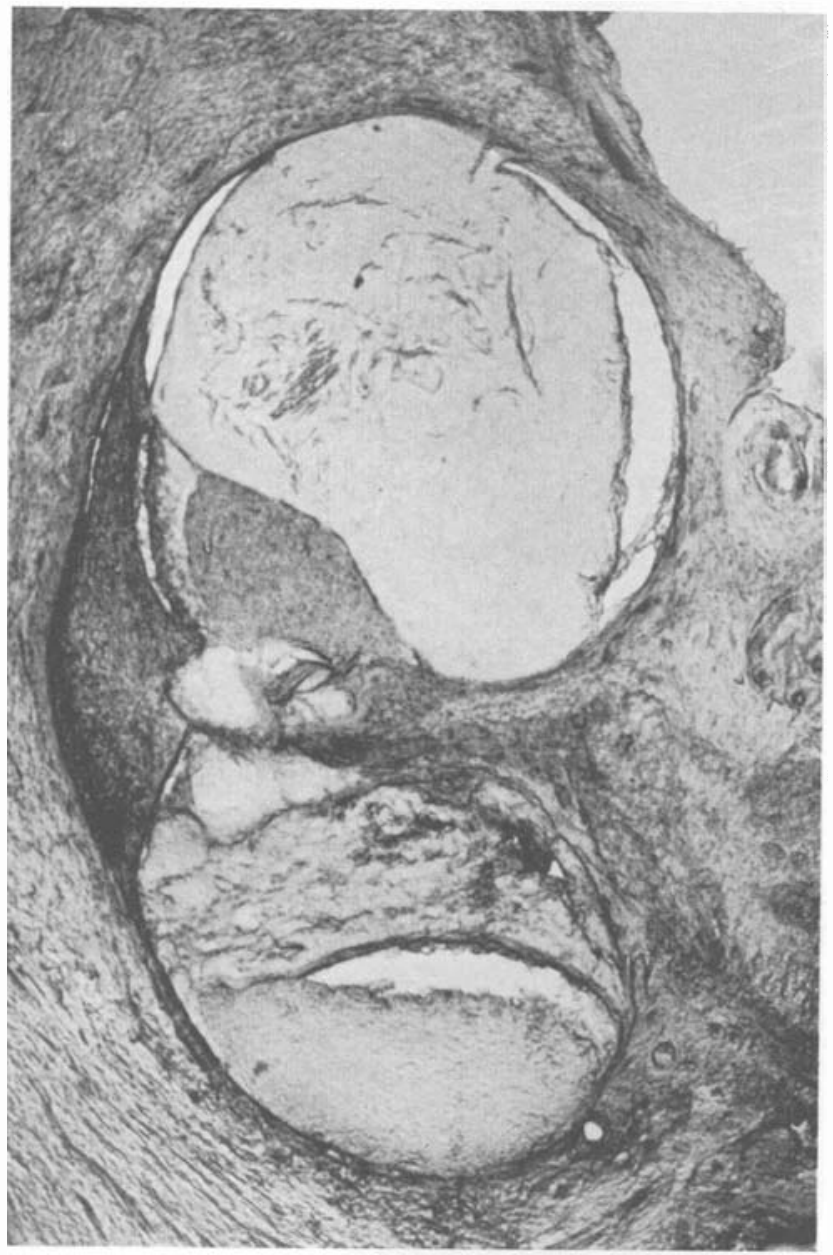

FIG. 5 .

Photomicrograph of the basal turn of the cochlea of Cat $4-72$.

\section{Cat $\mathbf{7} 7-72$.}

Bipolar stainless steel electrodes were inserted directly into the apical and basal turns. Penicillin was administered post-operatively, and the animal was perfused intra-arterially three months after implantation. 


\section{G. M. Clark et al.}

Histopathology (Fig. 6) showed marked destruction of the cochlea. There was loss of the organ of Corti in all turns. The scala vestibuli and tympani were filled with fibrous tissue, and the scala media with hyaline material. There was atrophy of about three-quarters of the spiral ganglion cells, and some degeneration of the auditory nerve.

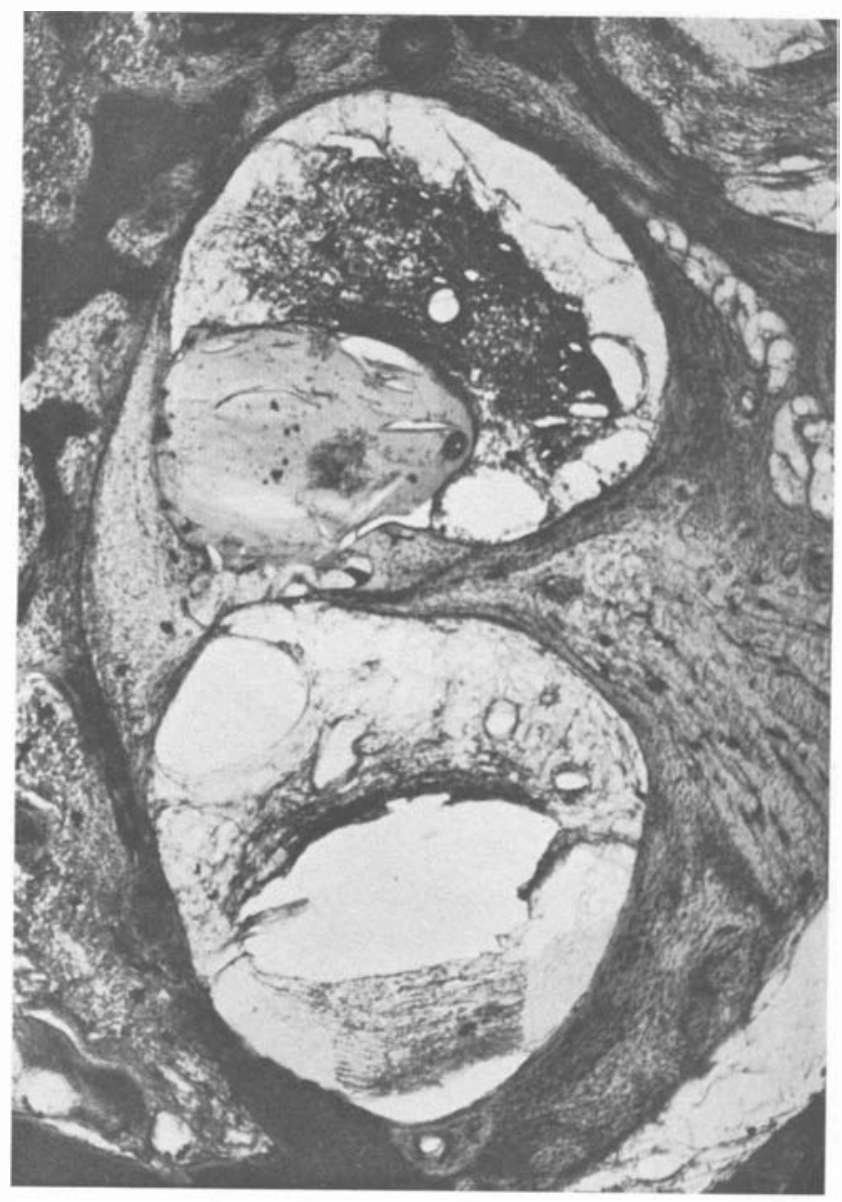

FIG. 6.

Photomicrograph of the cochlea of Cat $17-72$.

Cat $4-73$.

Bipolar silver electrodes were implanted directly into the basal turn following the previous implantation of a stainless steel electrode through the round window. Chloromycetin was administered post-operatively, and the animal was perfused intra-arterially three months after implantation.

Histopathology showed loss of the organ of Corti, and Reissner's membrane in all turns. There was a collection of polymorphs under the 


\section{Histopathological findings in cochlear implants in cats}

endothelial lining of the scala tympani of the basal turn. There were no spiral ganglion cells, and there was marked degeneration of the auditory nerve.

\section{Discussion}

The results of this study indicate that electrodes may be introduced into the cochlea through the round window or through an opening drilled in the bone overlying the middle and apical turns, without leading to a significant loss in the number of spiral ganglion cells or auditory nerve fibres. Furthermore, histopathological studies in man (Paparella and Sugiura, I967) have shown that following labyrinthitis secondary to meningitis there can still be significant numbers of spiral ganglion cells, and auditory nerve fibres even though the infection occurred many years previously and led to severe hearing loss and total loss of hair cells. Viral labyrinthitis (Lindsay, I973) and congenital deafness (Sugiura and Nomura, I970) can also result in the loss of hair cells, without an associated loss in spiral ganglion cells.

These reported studies indicate that it may be possible to use a cochlear implant for patients who have developed severe perceptive deafness many years previously from a variety of causes. They also showed that in many cases there was a greater loss of spiral ganglion cells in the basal rather than the middle and apical turns. Consequently, a cochlear implant should be placed in the middle and apical turns, and if this cannot be carried out by introducing electrodes through the round window, it may be done through an opening drilled over the middle and apical turns (Clark, I975).

Although the present experimental study has shown that direct implantation of electrodes into the cochlea can be done without leading to a loss in the spiral ganglion cells and auditory nerve fibres, it has also shown that there is a greater risk of infection using this surgical approach, and the resulting labyrinthitis can lead to serious destruction of the cochlear structures, and a marked loss of spiral ganglion cells. Consequently, if this form of experimental surgery is to be used, great care should be exercised in selecting patients to make sure that they do not have any useful hearing. This cannot be done from pure tone and speech audiograms alone, and the opinion of an educational audiologist should be obtained.

\section{Summary}

This histopathological study on cats has shown that electrodes may be implanted into the cochlea through the round window or an opening drilled over the middle and apical turns without loss of the spiral ganglion cells or auditory nerve fibres. It has also shown that infection, leading to 


\section{G. M. Clark et al.}

a labyrinthitis, was more likely to occur when an opening was drilled in the cochlea, and this could lead to a marked destruction of the cochlear structures, and loss of the spiral ganglion cells.

\section{Acknowledgements}

We would like to acknowledge the financial assistance provided by the Channel O Nerve Deafness Appeal, the Apex Club of Melbourne, the Felton Bequest, and the National Health and Medical Research Council of Australia.

We would also like to thank Mr. A. Duffet and Mr. P. Sommerville for technical assistance, Mrs. G. van den Brenk, and Miss C. Peterson from the Royal Victoria Eye and Ear Hospital for the photographic work, and Mrs. K. Hoogedeure for the typing.

\section{REFERENCES}

Axelsson, A., and Hallén, A. (1973) Acta Oto-Laryngologica, 76, 254.

CLARK, G. M. (I975) Journal of Laryngology and Otology. 89, 9.

Lindsay, J. R. (1973) Archives of Otolaryngology, 98, 258.

Paparella, M. M., and Sugiura, S. (1967) Annals of Otology, 76, 554.

Simmons, F. B. (1967) Laryngoscope, 77, i 7 I.

Sugiura, S., and Nomura, Y. (I970) Annals of Otology, 79, I I 39.

G. M. Clark,

Department of Otolaryngology,

University of Melbourne,

Parkville 3052 ,

Victoria,

Australia. 


\section{University Library}

\section{- M M I N E R VA A gateway to Melbourne's research publications}

Minerva Access is the Institutional Repository of The University of Melbourne

Author/s:

Clark, Graeme M.;Kranz, Howard G.;Minas, Harry;Nathar, J. M.

Title:

Histopathological findings in cochlear implants in cats

Date:

1975

Citation:

Clark, G. M., Kranz, H. G., Minas, H., \& Nathar, J. M. (1975). Histopathological findings in cochlear implants in cats. Journal of Laryngology and Otology, 89(5), 495-504.

Persistent Link:

http://hdl.handle.net/11343/27152 